\section{in}

https://www.linkedin.com/in/blanca-devega-s\%C3\%A1nchez-735776119/

\title{
An asymptomatic man with pathological chest radiography
}

\section{Case report}

A 76-year-old, male, ex-smoker (40 pack-years) was referred to our outpatient clinic for progressive dyspnoea (grade II-III/IV on the Medical Research Council scale [1]) over the last 20 years.

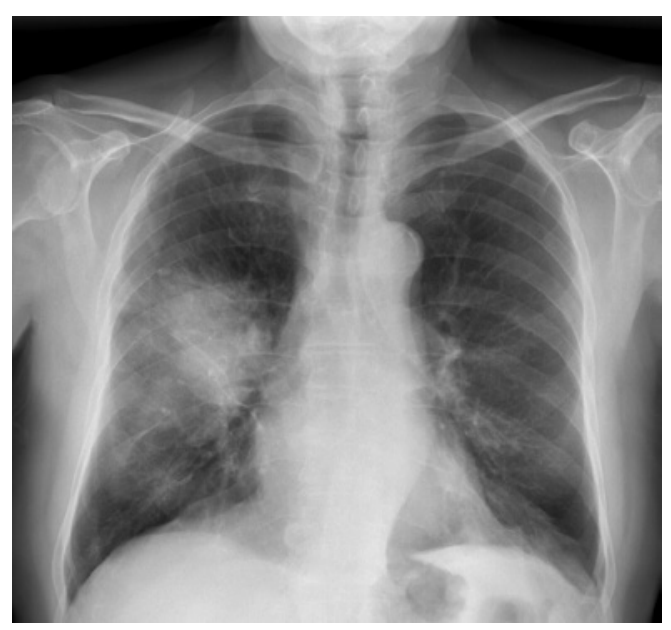

Figure 1 Chest radiograph.

\section{Task 1}

1. Describe the radiographic findings (figure 1).

a. Medial mediastinal enlargement with contralateral tracheal displacement

b. Bilateral interstitial pattern with multiple bilateral alveolar condensations predominating in the right hemithorax

c. Right hilar enlargement with middle lobe collapse

d. Small left unilateral pleural effusion with granuloma in the right upper lobe

2. Which of the following pathologies should not be included in the differential diagnosis?

a. Cryptogenic organising pneumonia

b. Pulmonary tuberculosis

c. Pleural elastofibroma

d. Pulmonary lymphoma
Cite as: De Vega Sanchez B, Disdier Vicente C, Lopez Pedreira MR. An asymptomatic man with pathological chest radiography. Breathe 2017; 13: e103-e108. 


\section{Answer 1}

1. b. Bilateral interstitial pattern with multiple bilateral alveolar condensations predominating in the right hemithorax. The other options were discarded; there was neither tracheal displacement (a), middle lobe collapse (c) nor pleural effusion (d).

2. c. Pleural elastofibroma. Pleural elastofibroma is an unusual neoplastic disease (estimated frequency of $5 \%$ of overall pleural tumours). Originating in the mesenchymal cells, it is usually found in the visceral pleura $(-80 \%)$, although it can be located in the parietal pleura, the mediastinum or other locations. It is typically benign ( $80 \%$ of cases) and it is often diagnosed as a chance finding due to its frequent asymptomatic behaviour.
The chest radiography (despite its limitations) performed in this patient did not seem to indicate pleural involvement. Taking into account the radiological findings and the patient's clinical history, the following should also be evaulated: sarcoidosis, advanced lung cancer disease and inflammatory pseudotumour.

\section{Task 2}

What further investigation would be most helpful?

a. Blood analysis with tumour markers

b. Complete pulmonary function test

c. Autoimmunity analysis

d. Complementary imaging tests (e.g. thoracic computerised axial tomography (CAT)) 


\section{Answer 2}

d. Complementary imaging tests. It would be most useful for the diagnosis to obtain complementary images, specifically CAT with contrast. This test allows the evaluation of parenchymal, bony and mediastinal alterations. This was performed and no abnormalities were found (figure 2).

Pulmonary function tests were within the limits of normality. Blood tests revealed weak positivity for antibodies (antinuclear, anti-DNA and anti-Jo) and normal tumour markers.
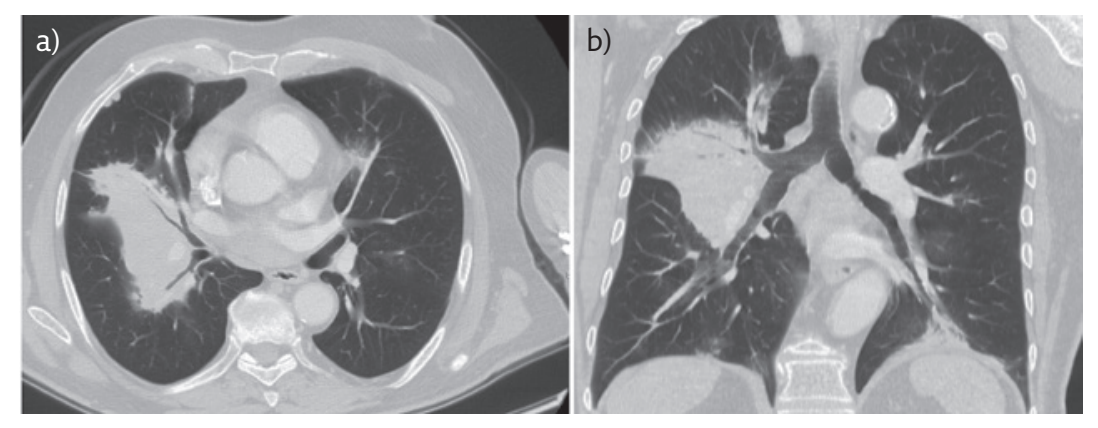

Figure 2 Thoracic CAT image. a) Axial. b) Coronal.

\section{Task 3}

Do you consider fibreoptic bronchoscopy mandatory?

a. Yes, this complementary test could provide more information and the possibility to perform other techniques.

b. No, the possibility of pulmonary neoplasia can be reasonably ruled out. The risk/ benefit ratio and the invasive nature of the technique would not justify it. 


\section{Answer 3}

a. Yes, this complementary test could provide more information and the possibility to perform other techniques. It is mandatory to perform flexible bronchoscopy because of the suspected diseases. The benefits of this technique are multiple: to discount unappreciable endobronchial alterations in the CAT scan (e.g. changes in the aspect of the bronchial mucosa suggestive of neoplastic formation), to perform microbiological sampling to rule out or confirm an infection, and to obtain of anatomopathological samples (e.g. bronchoalveolar lavage (BAL) and transbronchial biopsy). No endobronchial alterations were found except synechia in the upper right lobe (autofluorescence without pathological aspect).

The cellular analysis revealed the following.

- BAL: Iymphocytic alveolitis (35\%); $2 \%$ plasma cells and 3\% neutrophils

- Transbronchial biopsy: compatible with nonspecific inflammation

\section{Task 4}

1. In this case, what do you think is the best way to proceed (conservative versus invasive)?

a. The complementary tests performed so far allow a definitive diagnosis of lymphocytic pneumonia.

b. In the absence of a definitive diagnosis, to begin empirical glucocorticoid treatment is mandatory.

c. In the absence of a definitive diagnosis, a surgical biopsy/cryobiopsy is indicated.

d. Discuss with the patient the clinical approach to follow, offering the range of diagnostic and therapeutic possibilities.

2. Which of the following is the next step to take?

a. Multidisciplinary approach due to patient's age and characteristics.

b. Refer the patient to the thoracic surgery service for surgical biopsy. 


\section{Answer 4}

d. Discuss with the patient the clinical approach to follow, offering the range of diagnostic and therapeutic possibilities. After informing the patient of the available treatment options and the risk/ benefit balance, it was decided to begin treatment with systemic glucocorticoids (1 mg. kg $\left.{ }^{-1} \cdot \mathrm{day}^{-1}\right)$. However, there was neither clinical nor radiological improvement.

a. Multidisciplinary approach due to patient's age and characteristics. A multidisciplinary approach was taken involving the services of respiratory medicine, radiology and clinical pathology.

The suspicion of primary pulmonary lymphoma (the most frequent kind of lung lymphoma) arose after an in-depth radiological review (persistent bilateral alveolar consolidations in the right upper lobe, middle lobe and lower left lobe with slow growth) and the long-term evolution of the pathology. We explained to the patient the need to conduct a new biopsy owing to the absence of histological confirmation and the lack of response to glucocorticoid treatment. A new transbronchial biopsy was carried out but not before explaining the risks of the procedure. The pathology report concluded that there was lymphoid proliferation compatible with mucosaassociated B-lymphoma.

\section{Discussion}

Mucosa-associated lymphoid tissue (MALT) lymphoma is a rare entity, nevertheless representing the most common pulmonary B-cell lymphoma [2]. It was first described by ISAACSON and WRIGHT [3] in 1983 as an extranodal marginal zone lymphoma with small B-cells, monocytoid cells, small lymphocytes, and scattered immunoblast- and centroblast-like cells.

Despite its unknown origin, it has been associated with cytogenetic abnormalities (such as monoclonal gammopathy), Helicobacter pylori or Alcaligenes xylosoxidans infection, autoimmune diseases, antigenic stimulation (cigarette smoke and chronic inflammation), amyloid deposits, and AIDS [3-7].

Generally, only $50 \%$ of the patients are symptomatic, with nonspecific pulmonary symptoms: chronic cough, dyspnoea, chest pain and haemoptysis. The radiological alterations are indispensable to include this entity in the differential diagnosis. Either single or multiple nodules, or consolidation areas are the major radiographic patterns observed in patients suffering from pulmonary MALT lymphoma [8, 9]. In addition, there may also be ground-glass areas with dilated air bronchograms (bilateral or unilateral).

Differential diagnosis includes granulomatosis with polyangiitis (often accompanied by kidney failure and/or haemoptysis), sarcoidosis, perilymphatic metastatic disease spread (with lymphadenopathies on CAT), infections (more usually with fever and leukocytosis) such as bacterial pneumonia (slowly resolving/slow-growing organisms), organising pneumonia, pulmonary infarction and lepidic adenocarcinoma [2, 5].

The definitive diagnosis is based on histological analysis of the tumour tissue. Transbronchial biopsy has $88 \%$ sensitivity detecting MALT lymphoma. This patient's BAL showed an absence of tumour epithelial cells and pathogens. The presence of lymphocytic alveolitis can be also indicative of primary pulmonary lymphoma if the B-lymphocyte cell level is $>10 \%$ [2].

MALT lymphoma has a favourable prognosis (5-year survival rate of $90 \%[8,10])$. Current treatment options include surgery (if the lesion can be located), chemotherapy and immunotherapy (mostly using rituximab-chlorambucil therapy but no standard regimen has been established [6]; anti-CD20 monoclonal antibodies have a $70 \%$ response rate but a high disease recurrence rate [11]), antibiotics (no evidence of any pathogen after four courses of clarithromycin $2 \mathrm{~g}$ per $24 \mathrm{~h}$ for 12 days [6]), and radiotherapy (benefit of less morbidity). However, there is no evidence regarding the effectiveness of these treatments since no comparative group studies have been conducted. However, an expectant attitude might be the most suitable option in asymptomatic patients with limited disease. Either way, before deciding the therapeutic options, one should consider the patient's age, symptoms, dissemination and performance status [2].

\section{Conflict of interest}

None declared.

\section{References}

1. Fletcher CM, Clifton M, Fairbairn AS, et al. Standardised questionnaire on respiratory symptoms. BMJ 1960; 2 : 1665.
2. Borie R, Wislez M, Antoine, M, et al. Pulmonary mucosaassociated lymphoid tissue lymphoma revisited. Eur Respir J 2016; 47: 1244-1260. 
3. Isaacson P, Wright DH. Malignant lymphoma of mucosaassociated lymphoid tissue. A distinctive type of B-cell lymphoma. Cancer 1983; 52: 1410-1416.

4. Kurtin PJ, Myers JL, Adlakha $\mathrm{H}$, et al. Pathologic and clinical features of primary pulmonary extranodal marginal zone B-cell lymphoma of MALT type. Am J Surg Pathol 2001; 25 : 997-1008.

5. Sirajuddin A, Raparia K, Lewis VA, et al. Primary pulmonary lymphoid lesions: radiologic and pathologic findings. Radiographics 2016; 36: 53-70.

6. Bi L, LiJ, Dan W, et al. Pulmonary MALT lymphoma: a case report and review of the literature. Exp Ther Med 2015; 9: 147-150.

7. Sammassimo S, Pruneri G, Andreola G, et al. A retrospective international study on primary extranodal marginal zone lymphoma of the lung (BALT lymphoma) on behalf of
International Extranodal Lymphoma Study Group (IELSG). Hematol Oncol 2016; 34: 177-183.

8. Cadranel J, Wislez M, Antoine M. Primary pulmonary lymphoma. Eur Respir J 2002; 20: 750-762

9. Ahmed S, Kussick SJ, Siddiqui AK, et al. Bronchial-associated lymphoid tissue lymphoma: a clinical study of a rare disease. EurJ Cancer 2004; 40: 1320-1326.

10. Nicolson AG, Harris NL. Marginal zone B-cell lymphoma of the mucosaassociated lymphoid tissue (MALT) type. In: World Health Organization Classification of Tumours. Pathology and Genetics of Tumours of the Lung, Pleura, Thymus and Heart. Geneva, WHO Press, 2004; pp. 9699/3.

11. Bae YA, Lee KS, Han J, et al. Marginal zone B-cell lymphoma of bronchus-associated lymphoid tissue: imaging findings in 21 patients. Chest 2008; 133: 433-440. 